\title{
Copy Number Aberration Analysis to Predict Response to Neoadjuvant Anti-HER2 Therapy: Results from the NeoALTTO Phase III Clinical Trial
}

David Venet ${ }^{1}$, Mattia Rediti ${ }^{1}$, Marion Maetens ${ }^{1,2}$, Debora Fumagalli ${ }^{3}$, David N. Brown ${ }^{1,4}$, Samira Majjaj ${ }^{1}$, Roberto Salgado ${ }^{5,6}$, Lajos Pusztai ${ }^{7}$, Nadia Harbeck ${ }^{8}$, Sarra El-Abed ${ }^{3}$, Yingbo Wang ${ }^{9}$, Cristina Saura ${ }^{10}$, Henry Gomez ${ }^{11}$, Vladimir Fedorovich Semiglazov ${ }^{12}$, Evandro de Azambuja ${ }^{13}$, Jens Huober ${ }^{14,15}$, Paolo Nuciforo ${ }^{16}$, Serena Di Cosimo ${ }^{17}$, Martine Piccart ${ }^{18}$, Sherene Loi ${ }^{6}$, Françoise Rothé ${ }^{1}$, and Christos Sotiriou ${ }^{1}$

\section{ABSTRACT}

Purpose: The heterogeneity of response to anti-HER2 agents represents a major challenge in patients with HER2-positive breast cancer. To better understand the sensitivity and resistance to trastuzumab and lapatinib, we investigated the role of copy number aberrations (CNA) in predicting pathologic complete response (pCR) and survival outcomes in the NeoALTTO trial.

Experimental Design: The neoadjuvant phase III NeoALTTO trial enrolled 455 patients with HER2-positive early-stage breast cancer. DNA samples from 269 patients were assessed for genomewide copy number profiling. Recurrent CNAs were found with GISTIC2.0.

Results: CNA estimates were obtained for 184 patients included in NeoALTTO. Among those, matched transcriptome and wholeexome data were available for 154 and 181 patients, respectively. A significant association between gene copy number and $\mathrm{PCR}$ was

\section{Introduction}

During the last decade, treatment strategies combining chemotherapy and trastuzumab with either lapatinib, neratinib, or pertuzumab in early-stage HER2-positive breast cancer have been extensively studied (1-10). In particular, in neoadjuvant clinical trials, dual HER2 blockade with trastuzumab and lapatinib/pertuzumab improved rates of pathologic complete response (pCR) compared with the single HER2-targeting agents and chemotherapy $(1,2)$.

${ }^{1}$ Breast Cancer Translational Research Laboratory J.-C. Heuson, Institut Jules Bordet, Université Libre de Bruxelles (U.L.B.), Brussels, Belgium. ${ }^{2}$ Department of Oncology, Laboratory for Translational Breast Cancer Research, KU Leuven, Leuven, Belgium. ${ }^{3}$ Breast International Group (BIG), Brussels, Belgium. ${ }^{4}$ Center for Molecular Oncology, Memorial Sloan Kettering Cancer Center, New York, New York. ${ }^{5}$ Department of Pathology, GZA-ZNA Ziekenhuizen, Antwerp, Belgium. ${ }^{6}$ Division of Research, Peter MacCallum Cancer Centre, Melbourne, Australia. ${ }^{7}$ Breast Medical Oncology, Yale Cancer Center, Yale School of Medicine, New Haven, Connecticut. ${ }^{8}$ Breast Center, Dept OB\&GYN and CCC Munich, LMU University Hospital, Munich, Germany. ${ }^{9}$ Novartis Pharmaceuticals AG, Basel, Switzerland. ${ }^{10}$ SOLTI-Breast Cancer Research Group, Barcelona, Spain. "Department of Medical Oncology, Instituto Nacional de Enfermedades Neoplasicas, Lima, Peru. ${ }^{12}$ National Medical Research Center of Oncology named after N.N. Petrov, St. Petersburg, Russia. ${ }^{13}$ Institut Jules Bordet, Université Libre de Bruxelles (U.L.B.), Brussels, Belgium. ${ }^{14}$ Department of Obstetrics and Gynecology, University of Ulm, Ulm, Germany. ${ }^{15}$ Breast Center, Cantonal Hospital St Gallen, St Gallen, Switzerland. ${ }^{16}$ Molecular Oncology Group, Vall d'Hebron Institute of Oncology, Barcelona, Spain ${ }^{17}$ Department of Applied Research and Technological Development, Fondazione IRCCS Istituto Nazionale dei Tumori, demonstrated for ERBB2 amplification. Nevertheless, ERBB2 amplification ceased to be predictive once ERBB2 expression level was considered. GISTIC2.0 analysis revealed 159 recurrent CNA regions. Lower copy number levels of the 6q23-24 locus predicted absence of pCR in the whole cohort and in the estrogen receptorpositive subgroup. 6q23-24 deletion was significantly more frequent in TP53 wild-type (WT) compared with TP53-mutated, resulting in copy number levels significantly associated with lack of pCR only in the TP53 WT subgroup. Interestingly, a gene-ontology analysis highlighted several immune processes correlated to 6q23-24 copy number.

Conclusions: Our analysis identified $E R B B 2$ copy number as well as 6q23-24 CNAs as predictors of response to anti-HER2-based treatment. ERBB2 expression outperformed ERBB2 amplification. The complexity of the 6q23-24 region warrants further investigation.

However, response to treatment is heterogeneous, with approximately $40 \%$ to $60 \%$ of the patients with HER2-positive breast cancer presenting residual disease after neoadjuvant therapy. Beyond HER2 overexpression by IHC or amplification by ISH (11), multiple potential predictive biomarkers have been identified, including PI3K pathway activation (12-15), the truncated form of the HER2 receptor p95HER2 (16), serum levels of HER2 (17), tumor-infiltrating lymphocyte (TIL) levels (18), ESR1 and ERBB2 gene expression, HER2enriched PAM50 subtype, immune signatures $(6,19)$, as well as proliferation signatures (19), T-cell receptor (TCR) repertoire $(20)$

Milano, Italy. ${ }^{18}$ Department of Medical Oncology, Institut Jules Bordet, Université Libre de Bruxelles (U.L.B.), Brussels, Belgium.

Note: Supplementary data for this article are available at Clinical Cancer Research Online (http://clincancerres.aacrjournals.org/).

D. Venet and M. Rediti are the co-first authors of this article.

F. Rothé and C. Sotiriou are the co-last authors of this article.

Prior presentation: The results were partially presented at the 2017 San Antonio Breast Cancer Symposium, San Antonio, December 5-9, 2017.

Corresponding Author: Christos Sotiriou, Breast Cancer Translational Research Laboratory J.-C. Heuson, Department of Medical Oncology, Institut Jules Bordet, Université Libre de Bruxelles, Rue Héger Bordet 1, Brussels 1000, Belgium Phone: 32-2-541-34-28; E-mail: christos.sotiriou@bordet.be

Clin Cancer Res 2021;27:5607-18

doi: 10.1158/1078-0432.CCR-21-1317

(C)2021 American Association for Cancer Research 


\section{Translational Relevance}

Despite the identification of several biomarkers associated with treatment resistance in patients with HER2-positive breast cancer, response to treatments is heterogenous, highlighting the complexity of this disease. Here, we aim to identify novel biomarkers for predicting pathologic complete response (pCR) by performing an integrated analysis of copy number aberrations (CNA) and gene expression data from pretreatment HER2-positive tumor samples prospectively collected in the NeoALTTO trial. ERBB2 gene expression better predicted pCR compared with ERBB2 amplification, whereas lower copy number levels of 6q23-24 were associated with resistance to neoadjuvant HER2-targeted therapy. Our data suggest that 6q23-24 CNAs play a role in the response to antiHER2 therapy in the neoadjuvant setting, particularly in patients with estrogen receptor-positive tumors and/or wild-type TP53, warranting further exploration.

and circulating tumor DNA (21). In addition, differences in pCR rates according to hormone receptor status have been observed across several clinical trials (22), further highlighting the biological heterogeneity of these tumors. Nevertheless, HER2 positivity defined by IHC overexpression/ISH amplification remains the sole validated predictive biomarker (23).

To gain more insight into the mechanisms underlying resistance to anti-HER2 treatment, we assessed the copy number aberration (CNA) profiles of pretreatment tumor samples obtained from patients enrolled in the NeoALTTO clinical trial $(1,24,25)$, aiming to identify biomarkers associated with pCR and long-term outcomes.

\section{Materials and Methods}

\section{Patients and samples}

The Neoadjuvant Lapatinib and/or Trastuzumab Treatment Optimization [NeoALTTO, Breast International Group (BIG 106)] trial was a multicenter, phase III neoadjuvant clinical trial in which 455 patients with HER2-positive early-stage breast cancer were randomized to lapatinib $1,500 \mathrm{mg} /$ day (Arm A), weekly trastuzumab ( $4 \mathrm{mg} / \mathrm{kg}$ loading dose followed by $2 \mathrm{mg} / \mathrm{kg}$; Arm B), or the combination of lapatinib $1,000 \mathrm{mg} /$ day and trastuzumab (Arm C) for 6 weeks, followed by the addition of weekly paclitaxel $\left(80 \mathrm{mg} / \mathrm{m}^{2}\right)$ for further 12 weeks before surgery (lapatinib dose was reduced in combination with paclitaxel and trastuzumab). After surgery, patients were treated with three cycles of adjuvant chemotherapy (fluorouracil, epirubicin, and cyclophosphamide), followed by the same anti-HER2 treatment administered in the neoadjuvant phase to complete 1 year of treatment. The primary endpoint was the rate of pCR, defined as the absence of invasive tumor cells in the breast (ypT0/is). Event-free survival (EFS) and locoregional total $\mathrm{pCR}$, defined as the absence of invasive tumor cells in the breast and the ipsilateral axillary lymph nodes (ypT0/is ypN0), were secondary endpoints. Study design and trial results have been published previously $(1,24,25)$. The trial was approved by relevant ethics committees and health authorities at all participating sites. Written informed consent, including the participation to future biomarker research, was obtained from all participants. This study was approved by the TransALTTO committee and was conducted in accordance to the Declaration of Helsinki. Levels of TILs were available from previously published results (18). Gene expressions and PAM50 subtypes were obtained from RNA sequencing (RNAseq) as published previously (19). Mutation status of TP53 gene was retrieved from available exome sequencing data (14) as published previously (21).

\section{Copy number analysis}

DNA was extracted from baseline pretreatment core biopsies using the Qiagen DNeasy Blood and Tissue Kit according to the manufacturer's recommendations (Invitrogen). CNA profiling was performed using Affymetrix genome wide CytoScan HD arrays containing $2.75 \mathrm{M}$ probes covering 750,000 SNPs according to standard procedure. The median of absolute pairwise differences and Median AutoCorrelation across the $\log _{2}$ ratio intensities were used as quality control of the SNP arrays. Only informative probes displaying heterozygous genotype $(\mathrm{AB})$ and copy neutral state were kept for analysis. The $\log _{2}$ ratio intensities and B-allele frequency, grouped per patient, were segmented jointly using the multitrack segmentation algorithm in the $\mathrm{R}$ package copy number (ref. 26; version 1.6.0) to determine common breakpoints. Integer level estimates of total copy number and major allele were obtained using Genome Alteration Print (version 12/2012) as described (27). Regions significantly amplified/deleted were found with GISTIC2.0 (28). The genome instability index (GII) was defined as the fraction of genome altered (by gains/amplifications or losses/ deep deletions). The human genome version GRCh37/hg19 was used to reference cytoband and gene coordinates. Copy number levels obtained with GISTIC2.0 were normalized to a ploidy of 2 for further analyses. Copy number status was defined as "deep deletion" for $\mathrm{CN}$ values $\leq 0.7$, "loss" for $\mathrm{CN}$ values between 0.7 and 1.6, "neutral" when $>1.6$ and $\leq 2.5$, "gain" for $\mathrm{CN}$ values $>2.5$ and $\leq 5$, and "amplification" when $>5$.

Publicly available somatic mutation calls, HER2 and estrogen receptor (ER) status, and ASCAT segment files from the METABRIC study were retrieved from Pereira and colleagues (ref. 29; available at http://github.com/cclab-brca). Publicly available somatic mutations data, HER2/ER status information, and ASCAT segment files from the TCGA study were downloaded from the GDC platform at https:// portal.gdc.cancer.gov. Copy number data were analyzed applying the same procedure as for NeoALTTO data.

\section{Statistical analysis}

Logistic regressions on variance-normalized data, copy numbers being first log transformed, were used for analyses on pCR, whereas Cox regressions were used for survival analyses. In the univariate setting, Mann-Whitney tests were used to assess the significant association between a numerical data and a binary outcome, whereas Fisher exact tests were used to evaluate the association between categorical data. For survival analyses, $P$ values were derived from log rank tests. In the multivariate setting, $P$ values were obtained by using an ANOVA on nested logistic and Cox models. Multivariate analyses were performed adjusting for clinicopathological characteristics [age as continuous variable, ER status, tumor size (T2 vs. T3-4), nodal status (N0/1 vs. N2/3/X), grade (1/2/not available vs. 3 ), and treatment arm (combination vs. single arms)]. FDR were obtained using Benjamini and Hochberg method. Spearman rank test was used for correlation analyses. Gene ontology (GO) analyses were performed using topGO (RRID: SCR_014798; ref. 30). The R package ImSig (31) was used to evaluate a set of immune-related signatures describing immune cell subpopulations and biological processes. The study complies with the REMARK guidelines. 


\section{Results}

\section{CNA profiles in the NeoALTTO cohort}

We first characterized the CNA profiles of the pretreatment tumor biopsies from patients enrolled in the NeoALTTO trial. As shown in Fig. 1, samples from 269 out of the 455 patients enrolled in the trial were assessed using Affymetrix CytoScan HD arrays for genome-wide copy number profiling. High-quality profiles with detectable aberrations were obtained for $68 \%$ of the patients (184 of the 269 samples). No significant differences in patients' clinicopathologic characteristics were observed between the 184 cases with detectable CNAs and the whole NeoALTTO study (Supplementary Table S1). Of note, RNA-seq data, TIL levels, and whole-exome sequencing data were available for 154, 164, and 181 patients out of the 184 patients, respectively. All analyses were carried out using the whole $\mathrm{CN}$ cohort, except the analyses evaluating associations with $\mathrm{pCR}$ which were performed in the subgroup of 180 patients (97.8\%) with available locoregional total pCR status (ypT0/is ypN0).

The 85/269 (32\%) samples with no discernable CNAs which were excluded had a lower tumor content than the cohort with evaluable CNAs and were significantly enriched for stromal TILs compared with the NeoALTTO cohort with available TIL data $(P=0.022$; Supplementary Table S1), possibly explaining the lack of detectable CNAs. As shown in Fig. 2A, 36\% of the tumor samples were aneuploid, with 26\% being triploid and $9.8 \%$ being tetraploid or more. The aneuploidy level was not significantly associated with pCR nor EFS.

We then evaluated the impact of copy number levels on gene expression for the 154 patients with both CNA and gene expression profiles available. As illustrated in Fig. 2B, 38.7\% of the genes showed at least a correlation of $30 \%$ between the copy number and gene expression levels as witnessed by a Spearman $\rho>0.3$, whereas $2.4 \%$ showed a high correlation $(\rho>0.7)$ and ERBB2 gene being among the most cis-regulated genes $(\rho=0.75)$. FISH ratio values were available for 114/184 patients with $\mathrm{CN}$ data, and presented a correlation of 0.3 with $E R B B 2 \mathrm{CN}$ levels. This was in line with the correlation of 0.34 between ERBB2 gene expression levels and FISH ratio previously reported in NeoALTTO (19).

Of note, the distribution of the CNAs from the NeoALTTO samples was similar to those observed in HER2-positive patients from TCGA (32) and METABRIC (Fig. 2C-E; refs. 29, 33). In

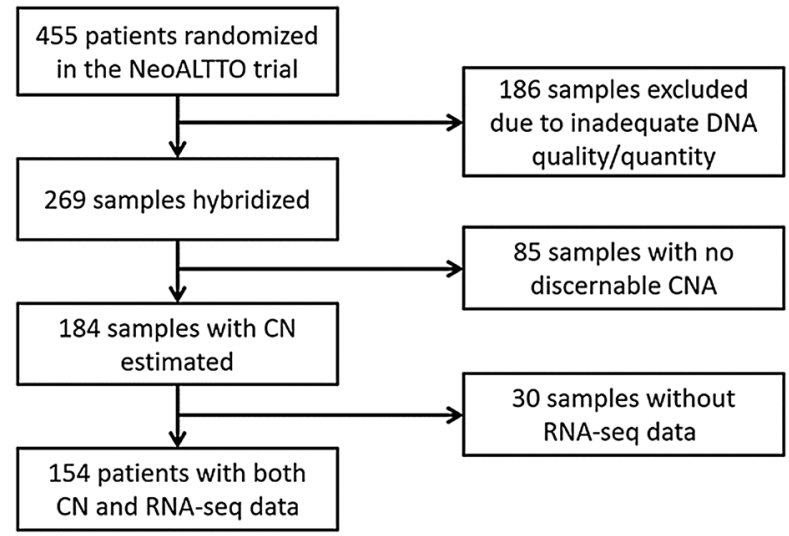

Figure 1.

CONSORT diagram of patient selection for secondary analysis in the NeoALTTO trial. Starting with 455 patients, 271 samples were removed due to inadequate DNA quality/quantity and absence of detectable CNA thus leaving 184 patients. Both copy number and RNA-seq data were available for 154 patients. particular, the Spearman correlation of the frequency of $\mathrm{CN}$ gains in NeoALTTO was 0.84 with TCGA and 0.86 with METABRIC. For $\mathrm{CN}$ losses, those correlations were 0.89 with TCGA and 0.95 with METABRIC.

\section{Differences between ER positive and ER negative among HER2-amplified tumors mirror those among HER2-negative tumors}

We next investigated whether the CNA profiles differed according to ER status in the NeoALTTO cohort. As illustrated in Fig. 3A, the frequencies of amplifications and deletions of many segments were significantly different between ER-positive and ER-negative tumor samples: for $8.8 \%$ of the genome, ER-positive and ER-negative tumors had significantly different CNA profiles $(F D R<0.01)$. These results were further validated using the HER2-positive samples from the TCGA dataset (Fig. 3B). We then evaluated whether the differences in CNA profiles observed between ER-positive and ER-negative tumors were specific to HER2-positive tumors or could be mirrored in HER2negative tumors as well (Fig. 3C). As shown in Fig. 3D-K, the difference of gains and deletions frequencies between ER-positive and ER-negative tumors were similar in HER2-positive and HER2-negative tumor samples from the TCGA (correlations of excess gains/ deletions in ER-positive/ER-negative between 0.45 and 0.68; Fig. 3D, E, $\mathbf{H}$, and I) and the METABRIC (correlations between 0.47 and 0.67; Fig. 3F, G, J, and K) datasets. However, these differences were about twice as much more frequent in the HER2-negative tumors as compared with HER2-positive tumors (slopes between 0.4 and 0.73 ). These data suggest that the CNA profiles are driven by ER rather than HER2 status.

\section{ERBB2 copy number level and gene expression predict $\mathrm{PCR}$}

We then evaluated whether copy number amplifications and/or deletions in specific cancer-related genes predicted response to neoadjuvant treatment. As ER status was shown to be a driver of CNAs, and is known to be a predictor of PCR, we corrected for ER status for this analysis. We first used a supervised approach investigating the association between CNAs located in specific breast cancer genes and $\mathrm{pCR}$ rate. Our analyses revealed that among the 49 genes known to harbor CNA in breast cancer from the COSMIC Cancer Gene Census database (Supplementary Table S2), $E R B B 2$ remained the only gene with $\mathrm{CN}$ levels significantly associated with pCR after multiple testing $(P<0.001 ; \mathrm{FDR}=0.02)$. As shown in Fig. 4A, ERBB2 CN was significantly associated to $\mathrm{pCR}$ in the whole population $[\mathrm{OR}=2.1 ; 95 \%$ confidence interval $(\mathrm{CI}), 1.4-$ 3.3; $P<0.001$, Mann-Whitney test $]$ as well as in ER-positive $(\mathrm{OR}=$ 3.5; 95\% CI, 1.4-8.8; $P=0.004)$ and, to a lesser extent, in ERnegative tumors $(\mathrm{OR}=1.6 ; 95 \% \mathrm{CI}, 1-2.6 ; P=0.023)$. Moreover, $E R B B 2 \mathrm{CN}$ levels were significantly higher in ER-negative tumors as compared with ER-positive ones $(P=0.016)$. Of interest, we also observed an association between $\mathrm{pCR}$ and FISH ratio $(\mathrm{OR}=1.7$; 95\% CI, 1.1-2.6; $P=0.03$ ), although less pronounced than the one with ERBB2 CN levels (OR $=2$; 95\% CI, 1.2-3.5; $P=0.0028$; analysis limited to the 114 patients with both FISH and CN data available). After correcting for clinicopathologic characteristics and treatment arm (Fig. 5A), the association of $E R B B 2 \mathrm{CN}$ levels with $\mathrm{pCR}$ remained significant in the whole population $(\mathrm{OR}=1.9 ; 95 \%$ $\mathrm{CI}, 1.2-3 ; P=0.003)$ as well as in ER-positive tumors $(\mathrm{OR}=4.6$; 95\% CI, 1.3-16; $P=0.0025)$. However, when ERBB2 gene expression level was taken into consideration in a multivariate analysis, $E R B B 2$ amplification was no longer predictive of pCR $(P=0.263)$. In contrast, $E R B B 2$ gene expression level still predicted pCR after 
A
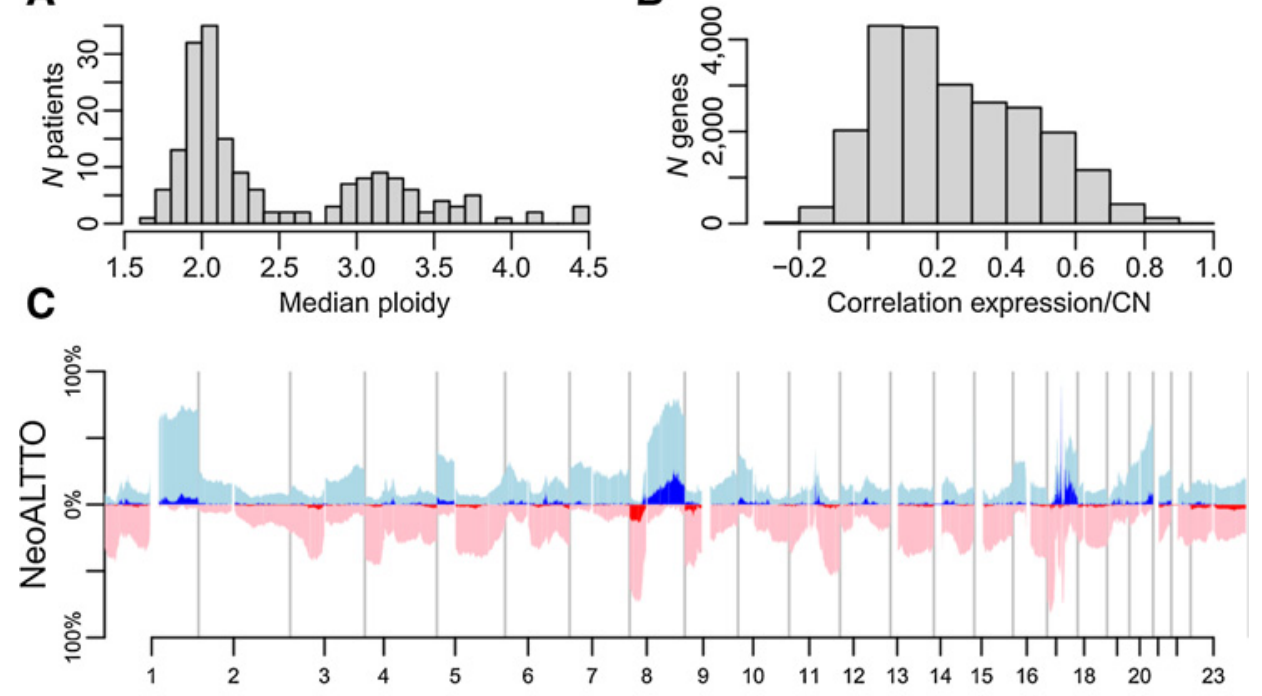

D

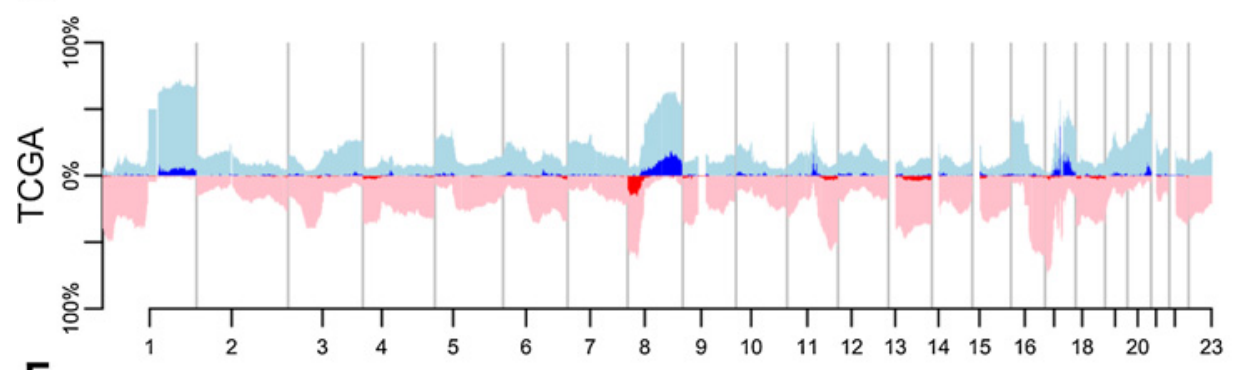

$E$

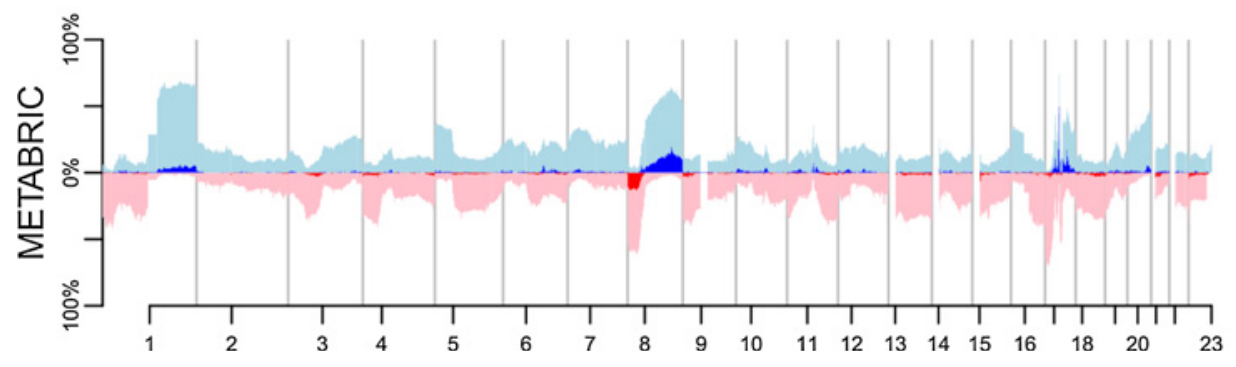

B

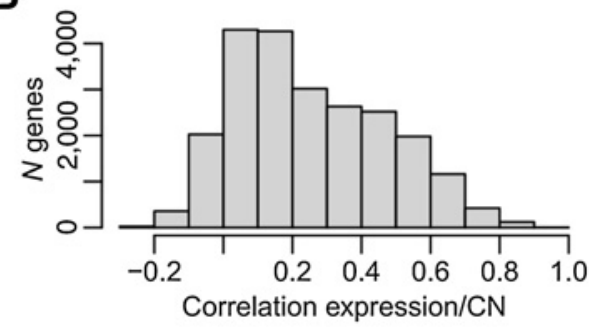

Figure 2.

Global statistics on the copy number profiles. A, Histogram of the ploidy estimated as the median copy number. B, Histogram of the correlation between gene copy number (CN) and associated mRNA gene expression. C-E, Comparison of the pattern of CNAs obtained on NeoALTTO, TCGA, and METABRIC. CN losses are shown in red and gains in blue (darker colors are used for deep deletions and amplifications, respectively).

ERBB2 amplification correction $(P=0.001)$, therefore suggesting that $E R B B 2$ gene expression predicted pCR better than ERBB2 amplification level. Furthermore, although no association was found between ERBB2 CN levels and EFS (Fig. 4B and 5B), we observed a significant association with overall survival (OS) in the ER-negative group at the univariate $(\mathrm{HR}=0.58 ; 95 \% \mathrm{CI}, 0.39-0.86$; $P=0.011)$ and multivariate $(\mathrm{HR}=0.53 ; 95 \% \mathrm{CI}, 0.33-0.83 ; P=$ 0.008 ) analyses, as depicted in Figs. 4C and 5C.

\section{GII and association with clinical outcomes}

Similarly, we evaluated whether GII as defined by the fraction of genome altered could predict pCR. We found no significant association between GII and pCR neither in the whole cohort nor according to ER status (Figs. 4A and 5A). With regards to survival outcomes, there was no significant association between the GII and EFS (Figs. 4B and 5B), although a significant association was found with OS in the whole population ( $\mathrm{HR}=0.61 ; 95 \% \mathrm{CI}, 0.4-0.93 ; P=0.019)$ as well as in the ER-positive subgroup ( $\mathrm{HR}=0.47 ; 95 \% \mathrm{CI}, 0.21-1 ; P=0.04)$ as depicted in Fig. 4C. These associations remained significant adjusting for clinicopathologic parameters (Fig. 5C). In addition, GII levels did not differ significantly when comparing ER-positive and ER-negative tumors $(P=0.086)$. Of note, a GO analysis of the genes correlated with the GII $(\rho>0.3)$ highlighted an enrichment in genes involved in cell proliferation (Supplementary Table S3), whereas a GO analysis on genes anticorrelated with GII $(\rho<-0.3)$ showed a prevalence of GO terms related to cellular processes (Supplementary Table S4).

\section{Chromosome 6q23-24 copy number levels are predictive of PCR}

We then investigated the regions of the genome that are significantly amplified or deleted across the tumor samples using GISTIC2.0 tool. We identified 159 recurrent regions with CNAs including 90 amplified and 69 deleted genomic regions totaling 1,093 Mbases (Mb). Of interest, many common oncogenes were included in these regions, 

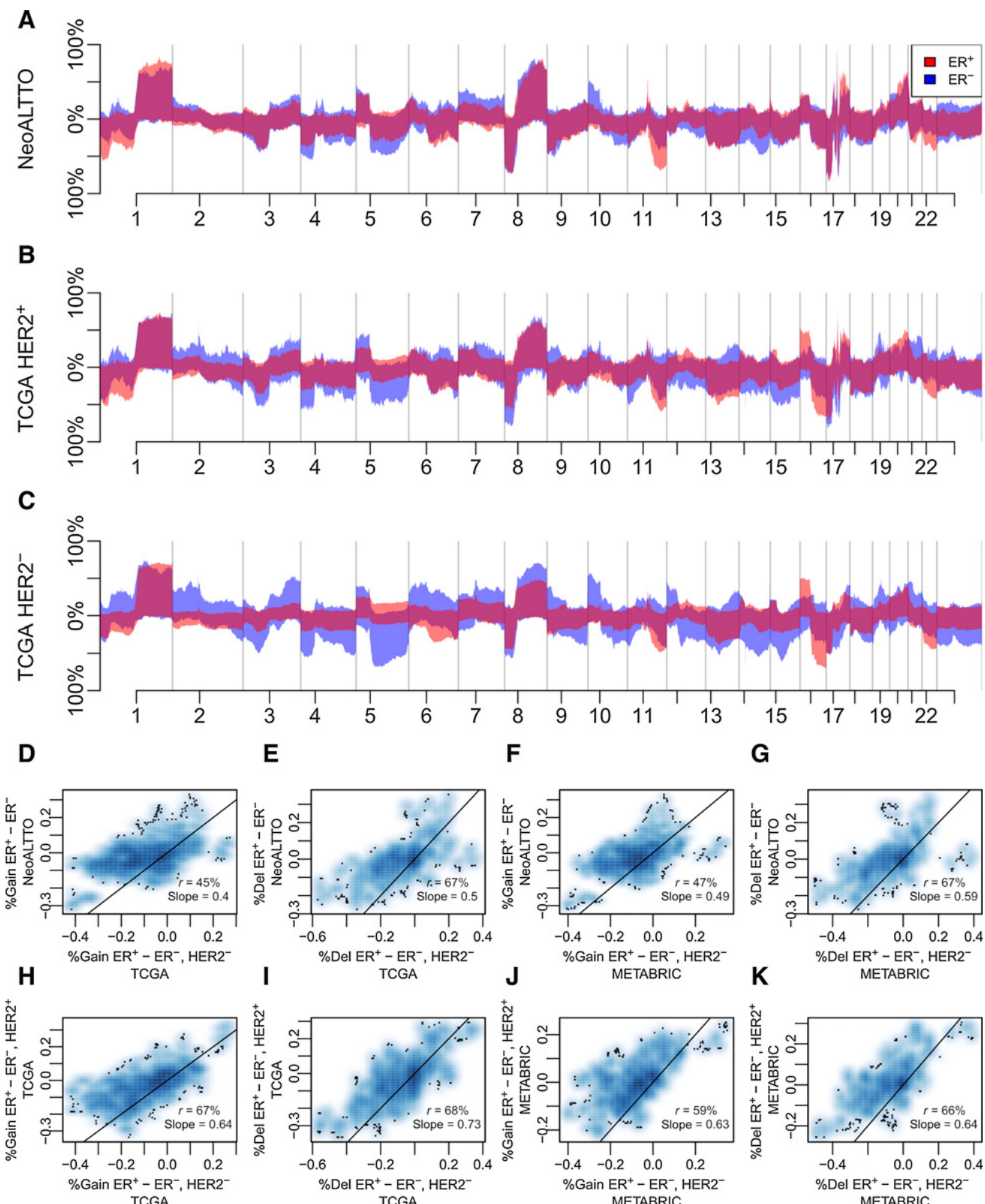

$\mathbf{F}$

G
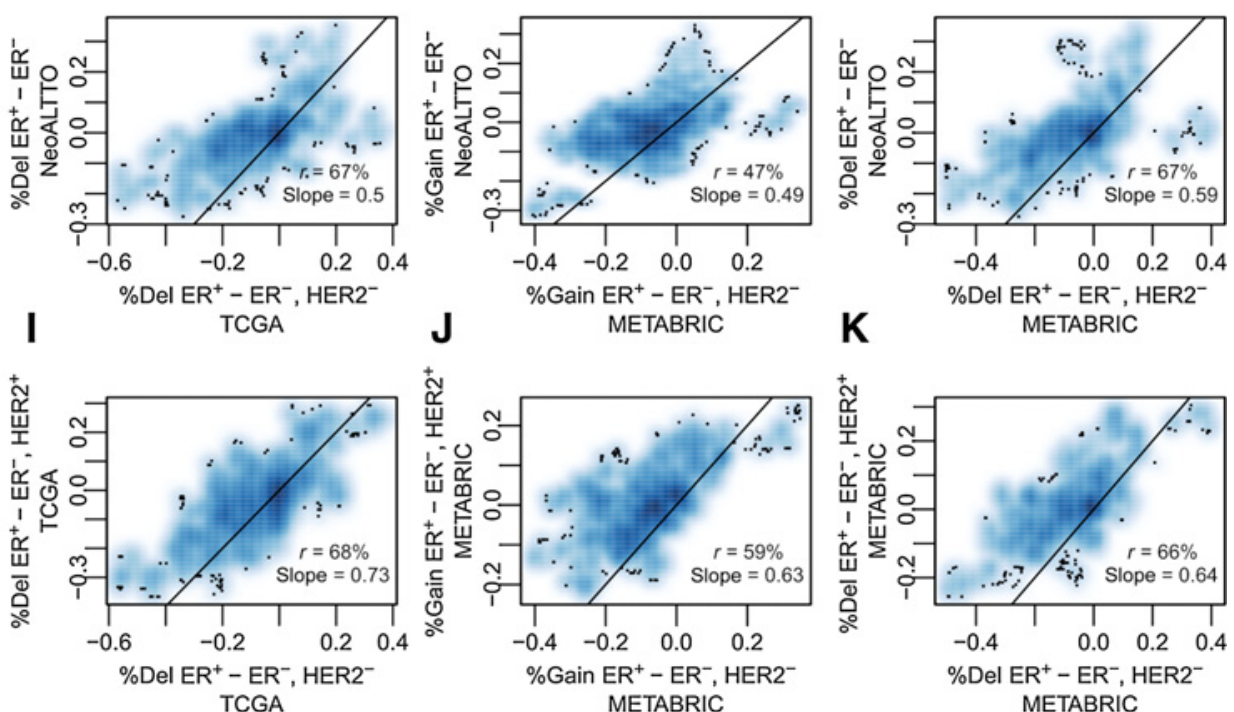

Figure 3.

Differences in copy number profiles according to estrogen receptor (ER) status. A-C, Comparison of the frequencies of amplifications (top) and deletions (bottom) in $\mathrm{ER}^{+}$(red) and ER ${ }^{-}$(blue) tumors in NeoALTTO, HER2 ${ }^{+}$patients in TCGA, as well as HER2 ${ }^{-}$patients in TCGA. D-K, Differences in the frequencies of amplification $(\mathbf{D}, \mathbf{F}, \mathbf{H}, \mathbf{J})$ or deletions $(\mathbf{E}, \mathbf{G}, \mathbf{I}, \mathbf{K})$ detected in $\mathrm{ER}^{+}$and $\mathrm{ER}^{-}$tumors between the HER2 ${ }^{+}$and $\mathrm{HER}^{-}$groups. Comparisons of $\mathrm{HER}^{-}$tumors in TCGA with HER2 ${ }^{+}$tumors in NeoALTTO ( $\mathbf{D}$ and $\mathbf{E}$ ) or TCGA (H and $\mathbf{I}$ ). Comparisons of HER2 ${ }^{-}$tumors in METABRIC with HER2 ${ }^{+}$tumors in NeoALTTO (F and $\left.\mathbf{G}\right)$ or METABRIC ( $\mathbf{J}$ and $\mathbf{K}$ ). Slopes are from orthogonal residuals fits. 
A

$\begin{array}{lr}\text { A } & \text { OR } \\ \text { ESR1 mRNA } & 0.49 \\ \text { ERBB2 mRNA } & 3.4 \\ \text { HER2-Enriched } & 4.3 \\ \text { ERBB2 CN All } & 2.1 \\ \text { ERBB2 CN ER } & 1.6 \\ \text { ERBB2 CN ER } & \\ \text { Gll All } & 3.5 \\ \text { Gll ER }^{-} & 1.2 \\ \text { Gll ER }^{+} & 0.98 \\ \text { 6q23-24 CN All } & 1.6 \\ \text { 6q23-24 CN ER } & 2.0 \\ \text { 6q23-24 CN ER } & 1.5 \\ & 3.3\end{array}$

B

$\begin{array}{lr} & \text { HR } \\ \text { ESR1 mRNA } & 0.85 \\ \text { ERBB2 mRNA } & 1.0 \\ \text { HER2-Enriched } & 1.3 \\ \text { ERBB2 CN All } & 0.91 \\ \text { ERBB2 CN ER } & 0.79 \\ \text { ERBB2 CN ER } & \\ \text { Gll All } & 1.1 \\ \text { Gll ER } & 0.78 \\ \text { Gll ER }^{+} & 0.81 \\ \text { 6q23-24 CN All } & 0.70 \\ \text { 6q23-24 CN ER } & 0.86 \\ \text { 6q23-24 CN ER } & 0.59 \\ & 1.2\end{array}$

C

$\begin{array}{lr} & \text { HR } \\ \text { ESR1 mRNA } & 0.71 \\ \text { ERBB2 mRNA } & 0.89 \\ \text { HER2-Enriched } & 1.3 \\ \text { ERBB2 CN All } & 0.77 \\ \text { ERBB2 CN ER } & 0.58 \\ \text { ERBB2 CN ER } & 1.3 \\ \text { Gll All } & 0.61 \\ \text { Gll ER } & 0.67 \\ \text { Gll ER } & \\ \text { 6q23-24 CN All } & 0.47 \\ \text { 6q23-24 CN ER } & 0.93 \\ \text { 6q23-24 CN ER } & 0.65 \\ & 1.3\end{array}$

$$
\begin{array}{r}
\mathrm{Cl} \\
0.32-0.74 \\
1.8-6.2 \\
2-9.3 \\
1.4-3.3 \\
1-2.6 \\
1.4-8.8 \\
0.88-1.7 \\
0.66-1.5 \\
0.92-2.9 \\
1.4-3 \\
0.98-2.4 \\
1.5-7.2
\end{array}
$$

Figure 4.

Association of single gene expression levels (ESR1 mRNA, ERBB2 mRNA), the HER2enriched PAM50 subtype, GII, and selected CNAs with clinical outcomes in the whole cohort and by estrogen receptor (ER) status. A, Forest plot for pCR (ypTO/is ypNO). $P$ values are from Mann-Whitney tests for numerical data (gene expression and copy number levels) and Fisher exact test for categorical data (HER2 enriched vs. other PAM50 subtypes). B, Forest plot for EFS C, Forest plot for OS. $P$ values for EFS and OS are from log-rank tests. 
Figure 5.

Association of single gene expression levels (ESRT mRNA, ERBB2 mRNA), the HER2-enriched PAM50 subtype, GII, and selected CNAs with clinical outcomes in the whole cohort and by estrogen receptor (ER) status, correcting for clinicopathologic parameters (age, ER status, tumor size, nodal status, grade, and treatment arm). A, Forest plot for pCR (ypTO/is ypNO). B, Forest plot for EFS. C, Forest plot for OS. $P$ values were obtained with an ANOVA on nested logistic and Cox models.

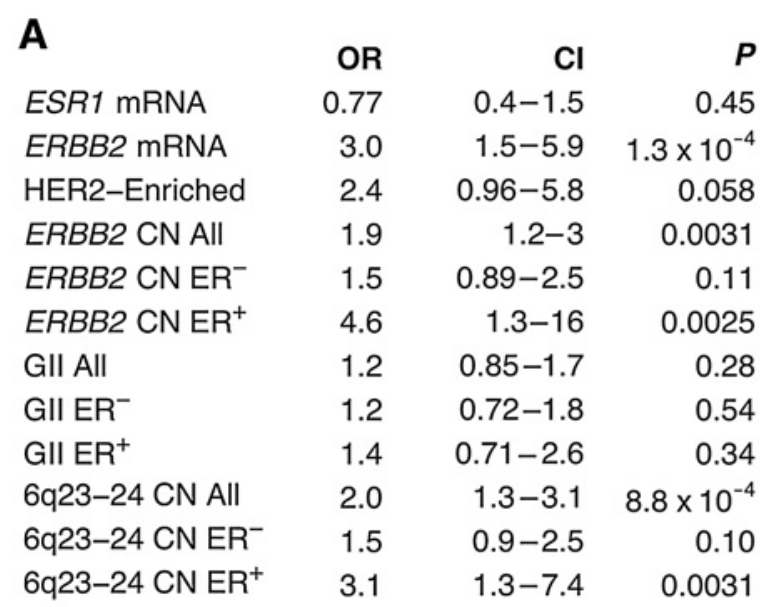

B

ESR1 mRNA
ERBB2 mRNA
HER2-Enriched
ERBB2 CN All
ERBB2 CN ER
ERBB2 CN ER
Gll All
Gll ER
GIl ER
6q23-24 CN All
6q23-24 CN ER-
6q23-24 CN ER ${ }^{+}$

C

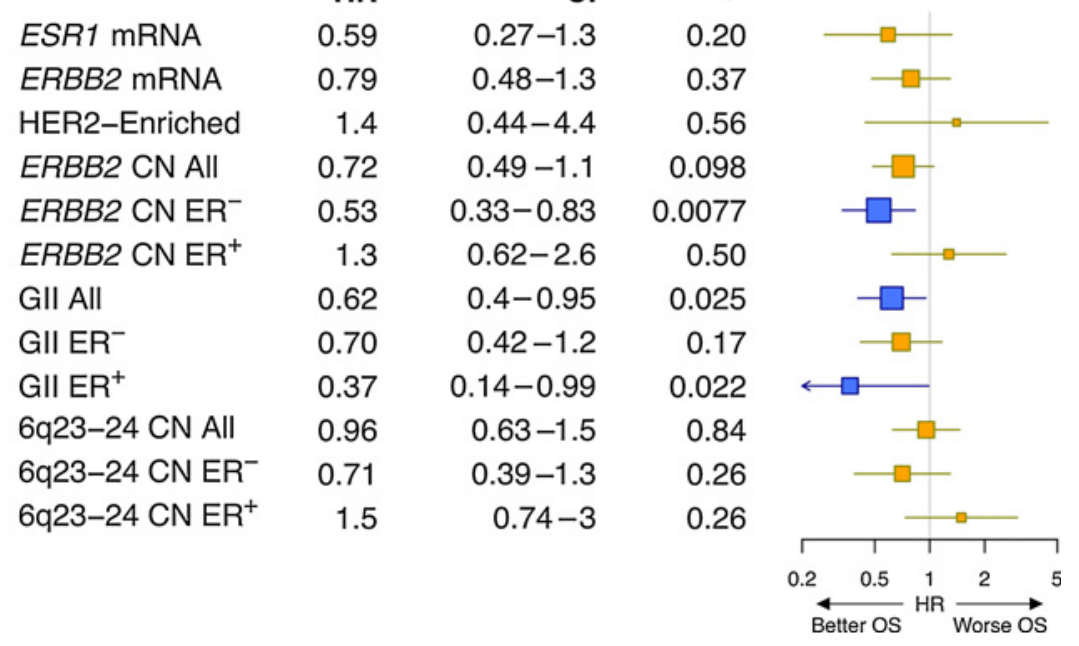


such as amplifications of ERBB2, PIK3CA, MYC, GATA3, and CCND1 genes and deletions of PTEN, ARID1A, RB1, and BRCA2 genes (Fig. 6A).

We next assessed whether specific regions pinpointed by GISTIC2.0 were associated with pCR (ypT0/is ypN0), again correcting for ER status. We identified two recurrent CNAs associated with $\mathrm{pCR}$ after correction for multiple testing: one region on chromosome 6q23-24 $(134172479-140617503 ; P<0.001 ; \mathrm{FDR}=0.038)$, and the region on $17 \mathrm{q} 12$ which includes the $E R B B 2$ gene $(P<0.001 ; \mathrm{FDR}=0.038)$.
Overall, we found one case with 6q23-24 deep deletion out of 184 patients with $\mathrm{CN}$ data $(0.5 \%), 33$ cases with $\mathrm{CN}$ loss $(17.9 \%), 32$ with $\mathrm{CN}$ gain (17.4\%), and 6 with $\mathrm{CN}$ amplification (3.3\%). The distribution of the 6q23-24 CNAs in the whole cohort and by ER status is shown in Fig. 6B.

Of interest, 6q23-24 CN levels were associated with the probability of achieving $\mathrm{pCR}$ in the whole population $(\mathrm{OR}=2 ; 95 \% \mathrm{CI}, 1.4-3 ; P<$ 0.001; Fig. 4A), with lower CN levels being associated to lower pCR rates (Fig. 6C). This association remained significant in the ER-
A
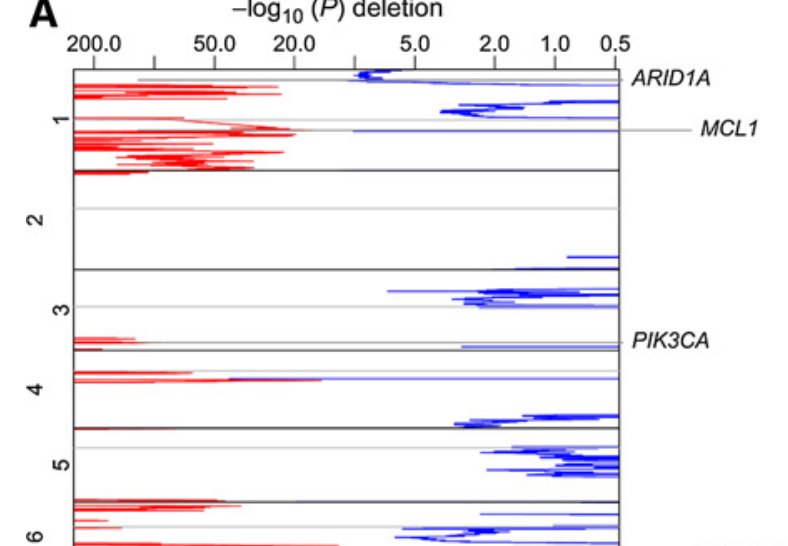

$\circ$

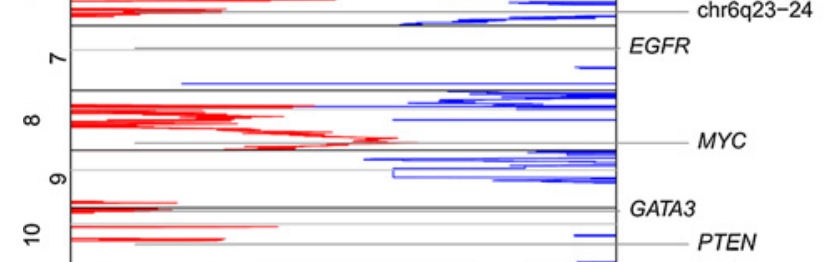

우

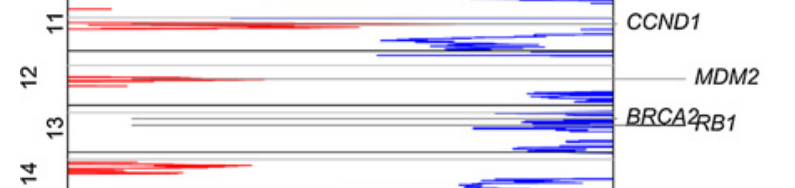

I

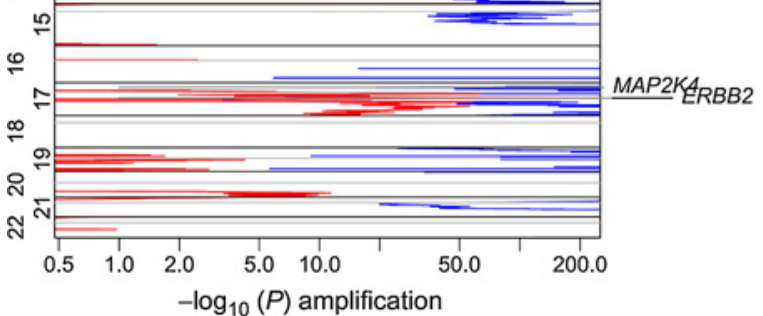

L

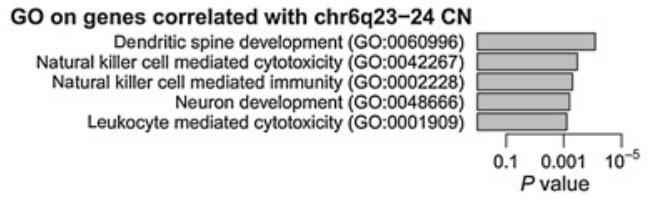

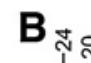

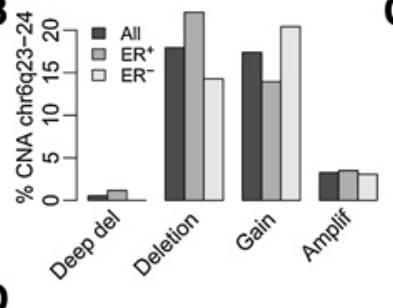

D

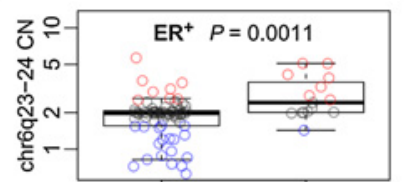

$\mathbf{F}$
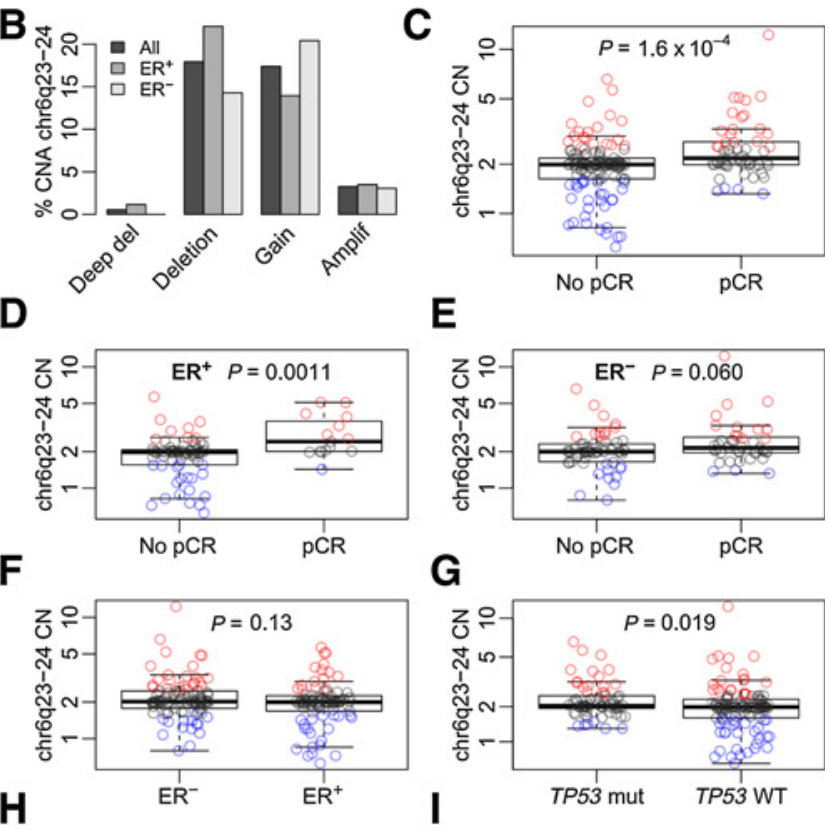

E

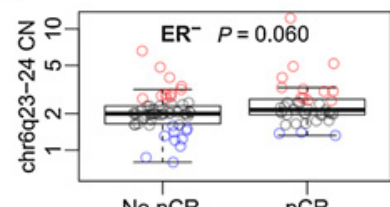

G
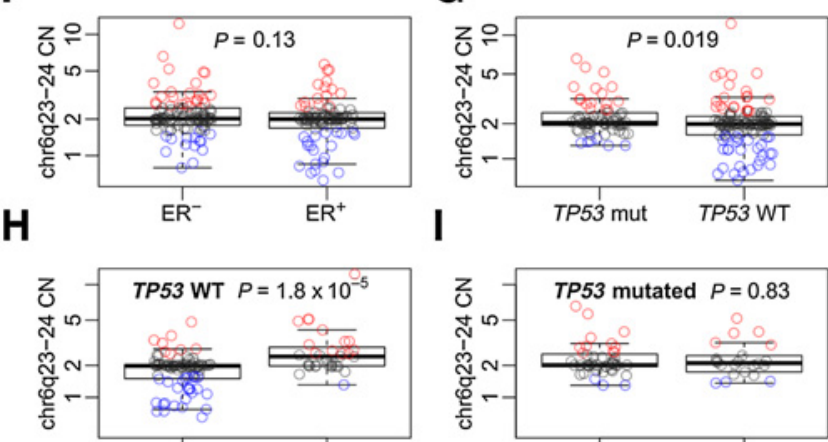

$\mathbf{J}$
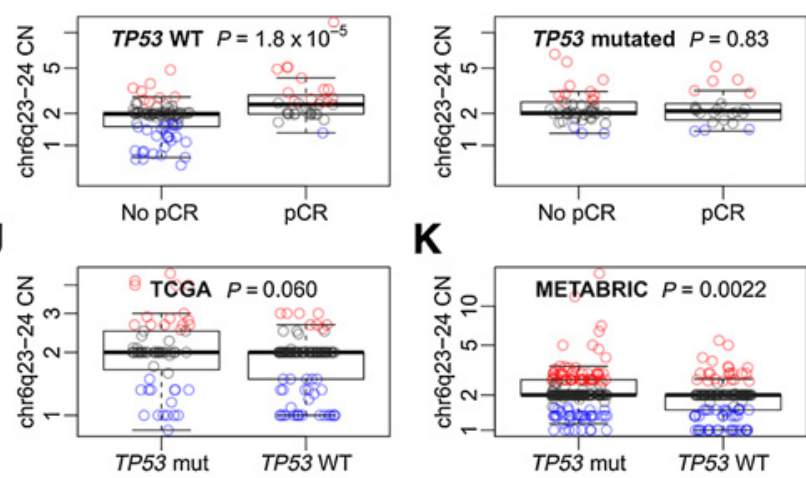

K
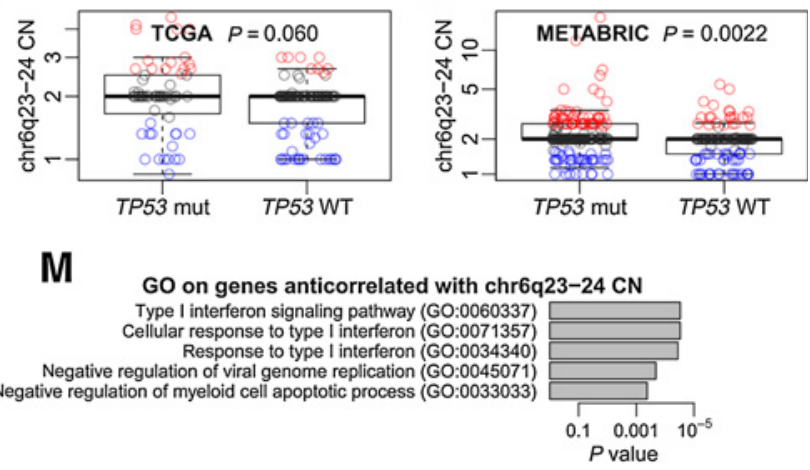

Figure 6.

Results from GISTIC analysis and association of the CNAs of 6q23-24 with pCR, estrogen receptor (ER) status, and TP53 mutation status. A gene ontology (GO) analysis highlighted several immune-related processes (anti-)correlated to chr6q23-24 copy number (CN) levels. A, Recurrent amplifications and deletions detected by GISTIC in relevant cancer-related genes (amplifications in red, deletions in blue). B, Frequency of the chr6q23-24 CN aberrations in the NeoALTTO cohort. C-F, Relationship between region chr6q23-24 CN and either pCR, ER status, or pCR by ER status (CN gain/amplification in red, CN loss/deep deletion in blue). G, Comparison between chr6q23-24 CN levels in TP53 wild-type (WT) and TP53-mutated tumors in NeoALTTO. $\mathbf{H}$ and $\mathbf{I}, \mathrm{pCR}$ in function of chr6q23-24 CN and TP53 status. J and $\mathbf{K}$, Comparisons of chr6q23-24 CN levels in TP53 WT and TP53-mutated tumors from TCGA and METABRIC. L, GO on genes correlated $(\rho>0.3)$ to chr6q23-24 CN levels. M, GO on genes anticorrelated ( $\rho<-0.3)$ to chr6q23-24 CN levels. The top five GO terms are shown. 
positive subgroup ( $\mathrm{OR}=3.3 ; 95 \% \mathrm{CI}, 1.5-7.2 ; P=0.001$; Figs. 4A and 6D), but not in ER-negative tumors, although the same trend was noted (Figs. 4A and 6E). Similar results were found after adjusting for clinicopathologic variables (Fig. 5A). Mutations in PIK3CA have been previously associated with lack of pCR in the NeoALTTO trial (14). In our cohort, PIK3CA was mutated in 48/181 (26.5\%) tumors with mutational data available. Of note, 6q23-24 $\mathrm{CN}$ levels remained significantly associated with $\mathrm{pCR}$ after controlling for PIK3CA mutational status $(\mathrm{OR}=2.2 ; 95 \% \mathrm{CI}, 1.4-3.3 ; P<0.001)$, as well as adjusting for clinicopathologic characteristics in addition to PIK3CA mutational status $(\mathrm{OR}=2.1 ; 95 \% \mathrm{CI}, 1.3-3.3 ; P<0.001)$. When evaluated as categorical variable, the $\mathrm{pCR}$ rate was lower in patients with tumors carrying 6q23-24 loss/deep deletion $(N=4 / 32$ with pCR information, $12.5 \%$ ) as compared with cases without the deletion (i.e., CN neutral/ gain/amplification; $N=51 / 148$ with pCR information, $34.5 \%$; $P=$ 0.019 , Fisher exact test), whereas tumors characterized by $6 \mathrm{q} 23-$ 24 gain/amplification presented higher pCR rates $(19 / 38,50 \%)$ when compared with those with 6q23-24 neutral/loss/deep deletion (36/142, $25.4 \% ; P=0.005$, Fisher exact test).

With regards to survival outcomes, 6q23-24 CN levels were significantly associated with EFS only in the ER-negative subgroup (HR = 0.59; 95\% CI, 0.38-0.91; $P=0.015$; Fig. 4B). This association did not remain significant after adjusting for clinicopathologic parameters (Fig. 5B). We did not observe any significant associations with OS (Figs. 4C and 5C).

The results related to pCR suggest that 6q23-24 CN levels have an impact on the tumor phenotype and on the response to neoadjuvant treatment following a gene dosage effect, with an effect at either low or high CN levels for at least some of the genes in this region. The 6q23-24 region was $6.4 \mathrm{Mb}$ long and contained 41 annotated genes, listed in Supplementary Table S5 together with their correlation in cis with CN levels and their association with pCR. Indeed, the expression level of some of those genes also predicted $\mathrm{pCR}$, in particular MAP3K5 gene $(P<0.001)$. Because several genes contained in this region may function as tumor-suppressor genes and/or encode proteins previously reported to interact with $\mathrm{p} 53$, such as $P E R P(34,35), B C L A F 1(36,37)$, HECA $(38,39)$, MAP3K5 (40), TBPL1 (41), and TNFAIP3 (42), we explored the association between 6q23-24 CN levels and TP53 mutational status. In the 181 patients with both $\mathrm{CN}$ and mutation data available, mutations in TP53 were present in 41.2\% (40/97) and 31\% (26/84) of ER-negative and ER-positive cases, respectively. This numerical difference was not statistically significant $(P=0.17)$. Of note, 6q23-24 deletions (either $\mathrm{CN}$ loss or deep deletion) were significantly enriched in TP53 wild-type (WT) tumors (28/115 for TP53 WT vs. $6 / 66$ for TP53 mutated; $P=0.01$, Fisher exact test). Indeed, 6q23-24 CN levels were significantly higher in patients with tumors carrying TP53 mutations compared with those with TP53 WT $(P=0.019$; Fig. 6G). This finding was confirmed in the METABRIC dataset $(P=0.002$; Fig. $6 \mathrm{~K})$, whereas the same trend was observed in the TCGA cohort $(P=0.06$; Fig. $\mathbf{6 J})$.

Interestingly, lower 6q23-24 CN levels were significantly associated with lack of pCR only for TP53 WT tumors (Fig. 6H; $P=0.004$, interaction test), whereas no significant association was noted in the presence of TP53 mutations (Fig. 6I).

We also observed a trend for higher pCR rate in patients with mutations in TP53, although not statistically significant. Indeed, in the cohort with $\mathrm{CN}$, mutation, and $\mathrm{pCR}$ information available $(N=177)$, pCR rates were $28.6 \%(32 / 112)$ and $33.8 \%(22 / 65)$ for WT and mutated TP53, respectively ( $P=0.5$, Fisher exact test).

Of note, we did not observe significant differences in the levels of 6q23-24 CN according to ER status (Fig. 6F).
A GO analysis of the genes whose expression was correlated with 6q23-24 CN levels $(\rho>0.3)$ highlighted an enrichment of genes involved in natural killer (NK) and leukocyte-mediated cytotoxicity (Fig. 6L), whereas analysis of genes anticorrelated $(\rho<-0.3)$ showed an enrichment of genes involved in the response to type I IFN (Fig. 6M). Of interest, the significant genes related to the GO terms for NK and leukocyte-mediated cytotoxicity were RAET1E, RAET1G, $U L B P 1, U L B P 2, U L B P 3$, a group of ligands for the activating receptor NK group 2 member D (NKG2D), and STX7. Despite these findings, we did not observe significant associations between TIL levels and 6q23-24 CN levels. Moreover, an analysis performed with ImSig showed a significant anticorrelation between the pathway "IFN response" and 6q23-24 CN levels ( $\rho=-0.225)$, in line with the GO analysis, whereas no significant correlation was found for NK or other immune cell subtypes.

\section{Discussion}

In this study we explored the potential role of CNAs in predicting the response to neoadjuvant HER2-targeting treatments and their association with survival outcomes in the context of the phase III NeoALTTO trial.

Similarly to what was previously observed for gene expression (19), $E R B B 2$ amplification level was predictive of $\mathrm{pCR}$ in patients treated with chemotherapy in combination with anti-HER2 agents, but not EFS. However, ERBB2 gene expression was a better predictor of $\mathrm{pCR}$ than ERBB2 amplification. This could be explained either because obtaining an accurate estimate of CNAs for what are sometimes very short amplifications can be challenging, or because gene expression is the real driver of the response.

Despite not finding significant association with pCR for genomic instability evaluated through copy number analysis, we highlighted an enrichment in genes involved in cell-cycle correlated to GII, suggesting an association between proliferation and accumulation of copy number changes in line with previous reports (43). Furthermore, GII was associated with OS in the whole population and in the ER-positive subgroup.

Of note, we have shown for the first time that lower 6q23-24 CN levels were predictive of absence of $\mathrm{pCR}$, with particular regards to ERpositive and/or TP53 WT tumors. Interestingly, a tumor-suppressor activity has been described for the 6q23-24 locus in sporadic endocrine pancreatic tumors (44), as well as for the region 6q22-23 in primary central nervous system lymphomas (45), whereas a prognostic role has been shown for 6q23 in melanoma (46). Furthermore, the 6q23-27 deletion is among the most common deletions in salivary gland carcinomas (47), whereas on the other hand, the amplification of 6q23-24 has been described in liposarcoma (48).

In particular, several genes included in the 6q23-24 locus have shown a tumor-suppressor activity and/or an interplay with TP53 in suppressing tumor growth, such as $\operatorname{HECA}(38,39), \operatorname{PERP}(34,35)$, TBPL1 (41), and TCF21 $(49,50)$, although genes promoting tumor growth are encompassed in this region as well. Both a tumor suppressive and an oncogenic role have been previously described for MAP3K5 (40), BCLAF1 (36, 37), and TNFAIP3 (42). Among the abovementioned genes, expression levels were significantly associated with pCR for HECA, MAP3K5, TBPL1, TCF21, and TNFAIP3.

The relation between deletion at 6q23-24 and TP53 mutational status in the NeoALTTO trial further hints at the importance of this region as a tumor-suppressor locus. In particular, our findings indicate that the deletion at 6q23-24 is more frequent in TP53 WT tumors and lower $\mathrm{CN}$ levels were significantly associated with lack of $\mathrm{pCR}$ only for 
TP53 WT patients, hinting that the deletion of this locus may be relevant only in tumors not harboring TP53 mutations. However, the complexity of this locus requires further evaluation.

Furthermore, TP53 was mutated in $41 \%$ of ER-negative and $31 \%$ of ER-positive tumors. In this regard, it has to be noted that functional mutations in TP53 have been previously reported to be more frequent in HER2-positive/ER-negative tumors compared with HER2-positive/ ER-positive tumors (29). We also observed a trend toward higher pCR rate in TP53-mutated tumors, although the difference was not statistically significant.

Our observation of lack of pCR associated to lower 6q23-24 CN levels in the whole cohort and in particular in the ER-positive population, may contribute to explaining the mechanisms underlying the lower pCR rates observed in patients with hormone receptorpositive tumors compared to hormone receptor-negative breast cancers in the NeoALTTO trial (1) and across other clinical trials (22).

Of particular interest, a GO analysis highlighted the correlation of several biological processes correlated to immune response with the amplification of chr6q23-24. These data suggest that tumors harboring 6q23-24 deletions may have an impairment of NK- and T-cellmediated cytotoxicity, thus possibly contributing to the observed lower pCR rates in this group of patients. Indeed, the role of the immune system, and in particular of $\mathrm{T}$ and NK cells, in mediating the response to anti-HER2 treatments through several mechanisms [e.g., antibody-dependent cellular cytotoxicity (ADCC)], is well described (51). In this regard, the GO results point toward a possible role of the ligands of the activating receptor NKG2D, which is expressed on the surface of several types of immune cells including NK cells (52), rather than the presence of specific subtypes of immune cells in the tumor samples. The NKG2D ligands can be induced in cells by proliferation, malignant transformation, and other conditions related to cellular "stress" (52). In the presence of low 6q23-24 CN levels, the interaction between NKG2D and its ligands may be less effective due to their low expression levels, potentially impairing ADCC driven by HER2-targeting agents. NKG2D ligands can be regulated by transcriptional, translational, and posttranslational mechanisms as well, potentially leading to immune-escape mechanisms with complex interactions (52). In this regard, our results have to be considered hypothesis generating and need further validation. In our study, among the biomarkers tested we found an association with EFS only for 6q23-24 CN levels in ER-negative patients. This finding, however, does not remain significant after adjusting for clinicopathologic variables. The NeoALTTO trial was not originally powered to evaluate the difference between the treatment arms in terms of EFS, and therefore some of our findings could be weakened by the low statistical power, also considering that our study was conducted in a subset of the original sample size.

An integrated analysis of copy number and gene expression was also performed in the METABRIC (33) study. However, none of the HER2positive patients received trastuzumab, therefore no information regarding response to anti-HER2 treatment could be extrapolated. More recently, Tanioka and colleagues performed an integrated analysis of CNAs, somatic mutations, gene signatures, and clinical variables of 137 pretreatment tumor samples from the neoadjuvant CALGB 40601 trial, aimed at developing models to predict pCR (53). In this study, the authors evaluated on 536 predetermined cancerspecific segments frequently altered, as well as on 48 segments derived from chromosome arm-based values. Interestingly, in their cohort TP53 mutations and the gain of another region of chromosome 6 , that is chr6p12-21, were associated with higher pCR rates, whereas other $6 \mathrm{p}$ regions were frequently selected in Elastic Net models to predict pCR.
In their analysis, the region 6q23.3 (included in the 6q23-24 segment identified in NeoALTTO) was not significantly associated with pCR, whereas in our study we did not find a significant association with pCR for the chr6p12-21 region, nor the three $6 \mathrm{p}$ regions most frequently selected in the models. The differences in the findings observed in the two studies may be related to the different methodologies used to assess relevant CNAs and their association with $\mathrm{pCR}$, or possible differences in the study populations.

Our study has various strengths. It is an analysis performed on frozen tissue samples prospectively collected in the context of a randomized phase III trial. This strengthens the reliability of the obtained results. The distributions of CNAs in HER2-positive tumors from our cohort were similar to those of HER2-positive tumors in TCGA and METABRIC datasets, confirming the comparability of the data, also in regard to our finding of lower 6q23-24 CN levels in TP53 WT tumors.

On the other hand, CNAs were obtained for only $40 \%$ of the patients enrolled in the trial (184/455), mostly due to low cellularity, which is always a challenge when evaluating tumor biopsies rather than whole surgical specimens. As low cellularity is correlated with higher TIL levels, this may induce a form of selection bias. This has further resulted in reducing the statistical power of our analysis when testing the association with long-term outcome but has not prevented us from finding valuable pCR-associated biomarkers.

In conclusion, our analysis of the CNAs in the NeoALTTO trial allowed us to identify two determinants of sensitivity to treatment: ERBB2 copy number as well as recurrent CNAs of 6q23-24. ERBB2 expression was however found to outperform ERBB2 amplification. Interestingly, CNAs at the 6q23-24 locus were found to impact pCR mainly in ER-positive and/or TP53 WT tumors. The complexity of this region warrants further investigation.

\section{Authors' Disclosures}

D. Fumagalli reports grants from GSK and Novartis during the conduct of the study, as well as grants from Roche/Genentech, AstraZeneca, Pfizer, Servier, and Tesaro outside the submitted work. R. Salgado reports other support from Merck, as well as personal fees from Roche and BMS outside the submitted work. L. Puszta reports personal fees from Pfizer, AstraZeneca, Merck, Novartis, Bristol-Myer Squibb, Genentech, Seagen, Syndax, H3Bio, and Daiichi outside the submitted work N. Harbeck reports personal fees from AstraZeneca, Daiichi-Sankyo, Exact Sciences, Lilly, Novartis, Pfizer, Pierre Fabre, Roche, Sandoz/Hexal, and Seagen outside the submitted work. S. El-Abed reports grants from Novartis during the conduct of the study, as well as grants from Pfizer and Genentech-Roche outside the submitted work. Y. Wang reports other support from Novartis during the conduct of the study C. Saura reports personal fees and other support from AstraZeneca, Novartis, Pfizer, Daiichi Sankyo, Eisai, Pierre Fabre, Puma, Roche Farma, and Seagen, as well as personal fees from Exact Sciences, Exeter Pharma, F. Hoffmann - La Roche Ltd., MediTech, Merck Sharp \& Dohme, Philips, Sanofi-Aventis, and Zymeworks outside the submitted work. E. de Azambuja reports other support from Roche/GNE Novartis, GSK, Libbs, Seattle Genetics, Zodiac, Pierre Fabre, and Lilly outside the submitted work; E. de Azambuja also reports research grants from Roche/GNE AstraZeneca, GSK/Novartis, and Servier, as well as travel grants from Roche/GNE and GSK/Novartis. J. Huober reports grants and personal fees from Novartis, Lilly, and Hexal; grants, personal fees, and other support from Roche and Celgene; personal fees and other support from Pfizer; personal fees from AbbVie, AstraZeneca, MSD, and Eisai; and other support from Daiichi outside the submitted work. P. Nuciforo reports grants from Novartis during the conduct of the study, as well as personal fees from Novartis, Roche/Genentech, MSD Oncology, Bayer, and Targos outside the submitted work. S. Di Cosimo reports personal fees from Pierre-Fabre and Novartis Pharma outside the submitted work, as well as Investigation Grant of Associazione Italian Ricerca contro il Cancro (AIRC) 20774. M. Piccart reports grants and personal fees from AstraZeneca, Immunomedics, Lilly, Menarini, MSD, Novartis, Pfizer, and Roche-Genentech; personal fees from Odonate, Camel-IDS, Debiopharm, Radius, Seattle Genetics, Immutep, Seagen, and NBE Therapeutics; and grants from Servier and Synthon outside the submitted work. S. Loi reports research funding to institution 
from Novartis, Bristol Meyers Squibb, Merck, Puma Biotechnology, Eli Lilly, Nektar Therapeutics, AstraZeneca, Roche-Genentech, and Seattle Genetics; consultant (not compensated) from Seattle Genetics, Novartis, Bristol Meyers Squibb, Merck, AstraZeneca, and Roche-Genentech; consultant (paid to institution) from Aduro Biotech Novartis, GlaxoSmithKline, Roche-Genentech, AstraZeneca, Silverback Therapeutics, G1 Therapeutics, PUMA Biotechnologies, Pfizer, Gilead Therapeutics, Seattle Genetics, and Bristol Meyers Squibb outside the submitted work. S. Loi is also a scientific advisory board member of Akamara Therapeutics. C. Sotiriou reports personal fees from Seattle Genetics, Puma, Amgen, Merck \& Co. Inc., Eisai, Prime Oncology, and Exact Sciences, as well as non-financial support from Roche, Genentech, and Pfizer outside the submitted work. No disclosures were reported by the other authors.

\section{Authors' Contributions}

D. Venet: Conceptualization, data curation, software, formal analysis, investigation, visualization, methodology, writing-original draft, writing-review and editing, co-first author. M. Rediti: Conceptualization, data curation, formal analysis, investigation, visualization, methodology, writing-original draft, writing-review and editing, co-first author. M. Maetens: Project administration, writing-review and editing. D. Fumagalli: Project administration, writing-review and editing. D.N. Brown: Data curation, formal analysis, methodology, writing-review and editing. S. Majjaj: Resources, investigation, writing-review and editing. R. Salgado: Resources, investigation, writing-review and editing. L. Pusztai Conceptualization, resources, writing-review and editing. N. Harbeck: Resources, writing-review and editing. S. El-Abed: Project administration, writing-review and editing. Y. Wang: Writing-review and editing. C. Saura: Resources, writing-review and editing. H. Gomez: Resources, writing-review and editing. V.F. Semiglazov: Resources, writing-review and editing. E. de Azambuja: Resources, writing-review and editing. J. Huober: Resources, writing-review and editing. P. Nuciforo:

\section{References}

1. Baselga J, Bradbury I, Eidtmann H, Di Cosimo S, de Azambuja E, Aura C, et al Lapatinib with trastuzumab for HER2-positive early breast cancer (NeoALTTO): a randomised, open-label, multicentre, phase 3 trial. Lancet 2012;379:633-40

2. Gianni L, Pienkowski T, Im Y-H, Roman L, Tseng L-M, Liu M-C, et al. Efficacy and safety of neoadjuvant pertuzumab and trastuzumab in women with locally advanced, inflammatory, or early HER2-positive breast cancer (NeoSphere): a randomised multicentre, open-label, phase 2 trial. Lancet Oncol 2012;13:25-32.

3. Robidoux A, Tang G, Rastogi P, Geyer CE, Azar CA, Atkins JN, et al. Lapatinib as a component of neoadjuvant therapy for HER2-positive operable breast cance (NSABP protocol B-41): an open-label, randomised phase 3 trial. Lancet Oncol 2013;14:1183-92.

4. Schneeweiss A, Chia S, Hickish T, Harvey V, Eniu A, Hegg R, et al. Pertuzumab plus trastuzumab in combination with standard neoadjuvant anthracyclinecontaining and anthracycline-free chemotherapy regimens in patients with HER2-positive early breast cancer: a randomized phase II cardiac safety study (TRYPHAENA). Ann Oncol 2013;24:2278-84.

5. Piccart-Gebhart M, Holmes E, Baselga J, de Azambuja E, Dueck AC, Viale G, et al. Adjuvant lapatinib and trastuzumab for early human epidermal growth factor receptor 2-positive breast cancer: results from the randomized phase III adjuvant lapatinib and/or trastuzumab treatment optimization trial. J Clin Oncol 2016;34:1034-42.

6. Carey LA, Berry DA, Cirrincione CT, Barry WT, Pitcher BN, Harris LN, et al. Molecular heterogeneity and response to neoadjuvant human epidermal growth factor receptor 2 targeting in CALGB 40601, a randomized phase III trial of paclitaxel plus trastuzumab with or without lapatinib. J Clin Oncol 2016;34: 542-9.

7. von Minckwitz G, Procter M, de Azambuja E, Zardavas D, Benyunes M, Viale G, et al. Adjuvant pertuzumab and trastuzumab in early HER2-positive breast cancer. N Engl J Med 2017;377:122-31.

8. Chan A, Delaloge S, Holmes FA, Moy B, Iwata H, Harvey VJ, et al. Neratinib after trastuzumab-based adjuvant therapy in patients with HER2-positive breast cancer (ExteNET): a multicentre, randomised, double-blind, placebo-controlled, phase 3 trial. Lancet Oncol 2016;17:367-77.

9. Martin M, Holmes FA, Ejlertsen B, Delaloge S, Moy B, Iwata H, et al. Neratinib after trastuzumab-based adjuvant therapy in HER2-positive breast cancer
Resources, investigation, writing-review and editing. S. Di Cosimo: Resources, writing-review and editing. M. Piccart: Resources, writing-review and editing S. Loi: Conceptualization, resources, writing-review and editing. F. Rothé: Conceptualization, supervision, investigation, visualization, methodology, writing-origina draft, project administration, writing-review and editing, co-last author. C. Sotiriou: Conceptualization, resources, supervision, funding acquisition, investigation, visualization, methodology, writing-original draft, project administration, writing-review and editing, co-last author.

\section{Acknowledgments}

This work has been supported by the Fondation contre le cancer (2012/211), the Breast Cancer Research Foundation, Les Amis de Bordet, and the Belgian Fonds National de la Recherche Scientifique (F.R.S-FNRS). M. Rediti was supported by Télévie and the Belgian Fonds National de la Recherche Scientifique (F.R.S.-FNRS). R. Salgado was supported by a grant from the Breast Cancer Research Foundation (BCRF, Grant No. 17-194). S. Di Cosimo is the recipient of the Associazione Italiana Ricerca contro il Cancro (AIRC) IG 20774. S. Loi was supported by the National Breast Cancer Foundation of Australia Endowed Chair and the Breast Cancer Research Foundation. The conduct of the NeoALTTO study was funded by GlaxoSmithKline and later Novartis. The RNA-seq on which part of the analyses described in this manuscript are based was funded by GlaxoSmithKline.

The costs of publication of this article were defrayed in part by the payment of page charges. This article must therefore be hereby marked advertisement in accordance with 18 U.S.C. Section 1734 solely to indicate this fact.

Received April 15, 2021; revised June 29, 2021; accepted July 23, 2021; published first July 28, 2021

(ExteNET): 5-year analysis of a randomised, double-blind, placebo-controlled, phase 3 trial. Lancet Oncol 2017;18:1688-700.

10. Guarneri V, Frassoldati A, Bottini A, Cagossi K, Bisagni G, Sarti S, et al. Preoperative chemotherapy plus trastuzumab, lapatinib, or both in human epidermal growth factor receptor 2-positive operable breast cancer: results of the randomized phase II CHER-LOB study. J Clin Oncol 2012; 30:1989-95.

11. Rimawi MF, Schiff R, Osborne CK. Targeting HER2 for the treatment of breast cancer. Annu Rev Med 2015;66:111-28.

12. Loibl S, von Minckwitz G, Schneeweiss A, Paepke S, Lehmann A, Rezai M, et al. PIK3CA mutations are associated with lower rates of pathologic complete response to anti-human epidermal growth factor receptor 2 (HER2) therapy in primary HER2-overexpressing breast cancer. JCO 2014;32:3212-20.

13. Majewski IJ, Nuciforo P, Mittempergher L, Bosma AJ, Eidtmann H, Holmes E, et al. PIK3CA mutations are associated with decreased benefit to neoadjuvant human epidermal growth factor receptor 2-targeted therapies in breast cancer. J Clin Oncol 2015;33:1334-9.

14. Shi W, Jiang T, Nuciforo P, Hatzis C, Holmes E, Harbeck N, et al. Pathway level alterations rather than mutations in single genes predict response to HER2targeted therapies in the neo-ALTTO trial. Ann Oncol 2017;28:128-35.

15. Veeraraghavan J, De Angelis C, Mao R, Wang T, Herrera S, Pavlick AC, et al. A combinatorial biomarker predicts pathologic complete response to neoadjuvant lapatinib and trastuzumab without chemotherapy in patients with HER2+ breast cancer. Ann Oncol 2019;30:927-33.

16. Scaltriti M, Nuciforo P, Bradbury I, Sperinde J, Agbor-Tarh D, Campbell C, et al High HER2 expression correlates with response to the combination of lapatinib and trastuzumab. Clin Cancer Res 2015;21:569-76.

17. Witzel I, Loibl S, von Minckwitz G, Eidtmann H, Fehm T, Khandan F, et al. Predictive value of HER2 serum levels in patients treated with lapatinib or trastuzumab - a translational project in the neoadjuvant GeparQuinto trial. Br J Cancer 2012;107:956-60.

18. Salgado R, Denkert C, Campbell C, Savas P, Nuciforo P, Nucifero P, et al. Tumorinfiltrating lymphocytes and associations with pathological complete response and event-free survival in HER2-positive early-stage breast cancer treated with lapatinib and trastuzumab: a secondary analysis of the NeoALTTO trial. JAMA Oncol 2015;1:448-54. 
19. Fumagalli D, Venet D, Ignatiadis M, Azim HA, Maetens M, Rothé F, et al. RNA sequencing to predict response to neoadjuvant anti-HER2 therapy: a secondary analysis of the NeoALTTO randomized clinical trial. JAMA Oncol 2017;3:227-34

20. Powles RL, Redmond D, Sotiriou C, Loi S, Fumagalli D, Nuciforo P, et al Association of T-cell receptor repertoire use with response to combined trastuzumab-lapatinib treatment of HER2-positive breast cancer: secondary analysis of the NeoALTTO randomized clinical trial. JAMA Oncol 2018;4:e181564.

21. Rothé F, Silva MJ, Venet D, Campbell C, Bradburry I, Rouas G, et al. Circulating tumor DNA in HER2-amplified breast cancer: a translational research substudy of the NeoALTTO Phase III Trial. Clin Cancer Res 2019;25:3581-8.

22. Brandão M, Caparica R, Malorni L, Prat A, Carey LA, Piccart M. What is the real impact of estrogen receptor status on the prognosis and treatment of HER2-positive early breast cancer? Clin Cancer Res 2020;26:2783-8.

23. Gingras I, Gebhart G, de Azambuja E, Piccart-Gebhart M. HER2-positive breast cancer is lost in translation: time for patient-centered research. Nat Rev Clin Oncol 2017;14:669-81.

24. de Azambuja E, Holmes AP, Piccart-Gebhart M, Holmes E, Di Cosimo S, Swaby RF, et al. Lapatinib with trastuzumab for HER2-positive early breast cancer (NeoALTTO): survival outcomes of a randomised, open-label, multicentre, phase 3 trial and their association with pathological complete response. Lancet Oncol 2014;15:1137-46.

25. Huober J, Holmes E, Baselga J, de Azambuja E, Untch M, Fumagalli D, et al. Survival outcomes of the NeoALTTO study (BIG 1-06): updated results of a randomised multicenter phase III neoadjuvant clinical trial in patients with HER2-positive primary breast cancer. Eur J Cancer 2019;118:169-77.

26. Nilsen G, Liestøl K, Van Loo P, Vollan HKM, Eide MB, Rueda OM, et al Copynumber: Efficient algorithms for single- and multi-track copy number segmentation. BMC Genomics 2012;13:591.

27. Popova T, Manié E, Stoppa-Lyonnet D, Rigaill G, Barillot E, Stern MH. Genome Alteration Print (GAP): a tool to visualize and mine complex cancer genomic profiles obtained by SNP arrays. Genome Biology 2009;10:R128.

28. Mermel CH, Schumacher SE, Hill B, Meyerson ML, Beroukhim R, Getz G. GISTIC2.0 facilitates sensitive and confident localization of the targets of focal somatic copy-number alteration in human cancers. Genome Biol 2011; 12:R41.

29. Pereira B, Chin S-F, Rueda OM, Vollan H-KM, Provenzano E, Bardwell HA, et al. The somatic mutation profiles of 2,433 breast cancers refine their genomic and transcriptomic landscapes. Nature Communications 2016;7:11479.

30. Alexa A, Rahnenfuhrer J. topGO: enrichment analysis for gene ontology. R package version $2.30 .1 ; 2016$.

31. Nirmal AJ, Regan T, Shih BB, Hume DA, Sims AH, Freeman TC. Immune cell gene signatures for profiling the microenvironment of solid tumors. Cancer Immunol Res 2018;6:1388-400.

32. Cancer Genome Atlas Network. Comprehensive molecular portraits of human breast tumours. Nature 2012;490:61-70.

33. Curtis C, Shah SP, Chin S-F, Turashvili G, Rueda OM, Dunning MJ, et al. The genomic and transcriptomic architecture of 2,000 breast tumours reveals novel subgroups. Nature 2012;486:346-52.

34. Dusek RL, Bascom JL, Vogel H, Baron S, Borowsky AD, Bissell MJ, et al. Deficiency of the p53/p63 target Perp alters mammary gland homeostasis and promotes cancer. Breast Cancer Research 2012;14:R65.

35. Davies L, Spiller D, White MRH, Grierson I, Paraoan L. PERP expression stabilizes active $\mathrm{p} 53$ via modulation of p53-MDM2 interaction in uveal melanoma cells. Cell Death Dis 2011;2:e136.
36. Lee YY, Yu YB, Gunawardena HP, Xie L, Chen X. BCLAF1 is a radiation-induced $\mathrm{H} 2 \mathrm{AX}$-interacting partner involved in $\gamma \mathrm{H} 2 \mathrm{AX}$-mediated regulation of apoptosis and DNA repair. Cell Death Dis 2012;3:e359.

37. Fang L, Du WW, Lyu J, Dong J, Zhang C, Yang W, et al. Enhanced breast cancer progression by mutant p53 is inhibited by the circular RNA circ-Ccnb1. Cell Death Diff 2018;25:2195.

38. Dowejko A, Bauer R, Bauer K, Müller-Richter UDA, Reichert TE. The human HECA interacts with cyclins and CDKs to antagonize Wnt-mediated proliferation and chemoresistance of head and neck cancer cells. Exp Cell Res 2012;318 489-99.

39. Wang J, Gong L, Zhu S-J, Zhu Q, Yao L, Han X-J, et al. The human homolog of Drosophila headcase acts as a tumor suppressor through its blocking effect on the cell cycle in hepatocellular carcinoma. PLoS ONE 2015;10:e0137579.

40. Ryuno H, Naguro I, Kamiyama M. ASK family and cancer. Adv Biol Regul 2017; 66:72-84.

41. Maeda R, Tamashiro H, Takano K, Takahashi H, Suzuki H, Saito S, et al. TBP-like protein (TLP) disrupts the p53-MDM2 interaction and induces long-lasting p53 activation. J Biol Chem 2017;jbc.M116.763318.

42. Lee J-H, Jung SM, Yang K-M, Bae E, Ahn SG, Park JS, et al. A20 promotes metastasis of aggressive basal-like breast cancers through multimonoubiquitylation of Snail1. Nat Cell Biol 2017;19:1260-73.

43. Davoli T, Uno H, Wooten EC, Elledge SJ. Tumor aneuploidy correlates with markers of immune evasion and with reduced response to immunotherapy. Science 2017;355:6322.

44. Barghorn A, Speel EJM, Farspour B, Saremaslani P, Schmid S, Perren A, et al. Putative tumor suppressor loci at $6 \mathrm{q} 22$ and $6 \mathrm{q} 23-\mathrm{q} 24$ are involved in the malignant progression of sporadic endocrine pancreatic tumors. Am J Pathol 2001;158:1903-11.

45. Nakamura M, Kishi M, Sakaki T, Hashimoto H, Nakase H, Shimada K, et al. Novel tumor suppressor loci on 6q22-23 in primary central nervous system lymphomas. Cancer Res 2003;63:737-41.

46. North JP, Vetto JT, Murali R, White KP, White CR, Bastian BC. Assessment of copy number status of chromosomes 6 and 11 by FISH provides independent prognostic information in primary melanoma. Am J Surg Pathol 2011;35:1146-50.

47. Seethala RR. Histologic grading and prognostic biomarkers in salivary gland carcinomas. Adv Anat Pathol 2011;18:29-45.

48. Horvai AE, DeVries S, Roy R, O'Donnell RJ, Waldman F. Similarity in genetic alterations between paired well-differentiated and dedifferentiated components of dedifferentiated liposarcoma. Modern Pathology 2009;22: $1477-88$.

49. Dai Y, Duan H, Duan C, Zhou R, He Y, Tu Q, et al. Down-regulation of TCF21 by hypermethylation induces cell proliferation, migration and invasion in colorectal cancer. Biochem Biophys Res Commun 2016;469:430-6.

50. Wang J, Gao X, Wang $M$, Zhang J. Clinicopathological significance and biological role of TCF21 mRNA in breast cancer. Tumor Biol 2015; 36:8679-83.

51. Griguolo G, Pascual T, Dieci MV, Guarneri V, Prat A. Interaction of host immunity with HER2-targeted treatment and tumor heterogeneity in HER2positive breast cancer. J Immunother Cancer 2019;7:90.

52. Lanier LL. NKG2D receptor and its ligands in host defense. Cancer Immunol Res 2015;3:575-82.

53. Tanioka M, Fan C, Parker JS, Hoadley KA, Hu Z, Li Y, et al. Integrated analysis of RNA and DNA from the phase III trial CALGB 40601 identifies predictors of response to trastuzumab-based neoadjuvant chemotherapy in HER2-positive breast cancer. Clin Cancer Res 2018;24:5292-304. 